\title{
Performance engineering for the Lattice Boltzmann method on GPGPUs: Architectural requirements and performance results
}

\author{
J. Habich ${ }^{\mathrm{a}}$, C. Feichtinger ${ }^{\mathrm{b}}$, H. Köstler ${ }^{\mathrm{b}}$, G. Hager ${ }^{\mathrm{a}}$, G. Wellein ${ }^{\mathrm{a}, \mathrm{b}}$ \\ ${ }^{a}$ Erlangen Regional Computing Center, University of Erlangen-Nuremberg, Germany \\ ${ }^{b}$ Department for Computer Science, University of Erlangen-Nuremberg, Germany
}

\begin{abstract}
GPUs offer several times the floating point performance and memory bandwidth of current standard two socket CPU servers, e.g. NVIDIA C2070 vs. Intel Xeon Westmere X5650. The lattice Boltzmann method has been established as a flow solver in recent years and was one of the first flow solvers to be successfully ported and that performs well on GPUs. We demonstrate advanced optimization strategies for a D3Q19 lattice Boltzmann based incompressible flow solver for GPGPUs and CPUs based on NVIDIA CUDA and OpenCL. Since the implemented algorithm is limited by memory bandwidth, we concentrate on improving memory access. Basic data layout issues for optimal data access are explained and discussed. Furthermore, the algorithmic steps are rearranged to improve scattered access of the GPU memory. The importance of occupancy is discussed as well as optimization strategies to improve overall concurrency. We arrive at a well-optimized GPU kernel, which is integrated into a larger framework that can handle single phase fluid flow simulations as well as particle-laden flows. Our 3D LBM GPU implementation reaches up to 650 MLUPS in single precision and 290 MLUPS in double precision on an NVIDIA Tesla C2070.
\end{abstract}

Keywords: Parallelization, GPGPU, HPC, CUDA, OpenCL, Computational Fluid Dynamics, Lattice Boltzmann Method, Performance Modeling and Engineering

\section{Introduction}

Graphics Processing Units (GPUs) came a long way towards desk-side supercomputers. Despite the fate of other accelerators, they continue to thrive. Coming originally from the computer gaming sector, NVIDIA GPUs are now also marketed in a separate line called "Tesla", which meets the requirements of computing centers and HPC cluster operators. We evaluate the potential of the available NVIDIA CUDA GPU generations, namely G80, GT200 and GF100 (Fermi), for the kernel of a flow solver based on the lattice Boltzmann method (LBM) introduced in section 2 Early GPU adoptions of the LBM [1] were based on graphics APIs, which was a very tedious task. More recent research is focused on performance [2, 3] as well as more complex phenomena [4] than plain channel flow. This should give insight into

\footnotetext{
Email addresses:

Johannes.Habich@rrze.uni-erlangen.de (J. Habich), Christian.Feichtinger@informatik.uni-erlangen.de (C. Feichtinger), Harald.Koestler@informatik.uni-erlangen.de (H. Köstler), Georg.Hager@rrze.uni-erlangen.de (G. Hager), Gerhard.Wellein@rrze.uni-erlangen.de (G. Wellein)
}

peak performance attainable with contemporary hardware and show the sustainability of GPGPU computing. The risk of one single hardware vendor is indisputable for all applications and certainly limits sustainability in the long term. In contrast to CUDA, the Open Compute Language (OpenCL) is applicable to NVIDIA and AMD GPUs as well as CPUs and has gained interest as a unified programming paradigm in HPC. In section 3 we discuss general features of NVIDIA and AMD GPUs and suitable parallel programming paradigms and perform STREAM benchmarks. After that we briefly introduce the WaLBerla framework in section 5 . WaLBerla is a highly parallel software package for e.g. fluid simulation based on LBM, where we have integrated our optimized LBM GPU kernels as explained in section 6. In section 7 we evaluate the OpenCL performance on different GPUs in comparison to our CUDA kernel. This gives a comparison between maximum performance and a maximum variety of hardware platforms. 


\section{The lattice Boltzmann method}

The lattice Boltzmann method (LBM) is a versatile approach to solve incompressible flows based on a simplified gas-kinetic description of the Boltzmann equation [5, 6, 7, 8]. The LBM operates on a uniform grid of lattice nodes, which are updated with nearest neighbor information in every time step.

Based on a velocity discrete Boltzmann equation with an appropriate collision term, e.g. the BGK collision approximation, the LBM formulates as the following evolution equation:

$$
\begin{aligned}
f_{i}\left(\vec{x}+\vec{e}_{i} \delta t, t+\delta t\right)= & f_{i}(\vec{x}, t)- \\
& -\frac{1}{\tau}\left[f_{i}(\vec{x}, t)-f_{i}^{\mathrm{eq}}(\rho, \vec{u})\right] \\
& i=0 \ldots N .
\end{aligned}
$$

Particle distribution functions $f_{i}$ represent the probability of particles at position $\vec{x}$ and time step $t$ with the velocity $\vec{e}_{i} . \quad f_{i}^{\mathrm{eq}}$ is an approximation of the Maxwell-Boltzmann equilibrium distribution function at low Mach numbers and is solely based on the first moments of the particle distribution function (PDF), i.e. macroscopic fluid density $\rho$ and macroscopic fluid velocity $\vec{u}$. The shape of the numerical grid and the discrete velocity vectors $\vec{e}_{i}$ are derived from the discretization applied, i.e. the D3Q19 model [8], which uses 19 discrete velocities in 3-D.

To advance one timestep the following steps need to be taken for each cell of the domain:

- Compute the local macroscopic flow quantities $\rho$ and $\vec{u}$ from the distribution functions, $\rho=\sum_{i=0}^{N} f_{i}$ and $\vec{u}=\sum_{i=0}^{N} f_{i} \vec{e}_{i}$.

- Calculate the equilibrium distribution $f_{i}^{\text {eq }}$ from the macroscopic flow quantities (see [8] for the equation and parameters) and execute the "collision" (relaxation) process, $f_{i}^{*}\left(\vec{x}, t^{*}\right)=f_{i}(\vec{x}, t)-$ $\frac{1}{\tau}\left[f_{i}(\vec{x}, t)-f_{i}^{\mathrm{eq}}(\rho, \vec{u})\right]$, where the superscript “*” denotes the post-collision state ("collide step").

- Propagate the $i=0 \ldots N$ post-collision states $f_{i}^{*}\left(\vec{x}, t^{*}\right)$ to the appropriate neighboring cells according to the direction of $\vec{e}_{i}$, resulting in $f_{i}(\vec{x}+$ $\left.\vec{e}_{i} \delta t, t+\delta t\right)$, i.e., the values of the next timestep ("stream step").

For arbitrary domains with cells marked as non fluid, i.e. obstacles, a fourth step must be applied to handle the boundaries, the so-called "bounce-back" rule [9]. This step can be combined with the third step, the streaming step, as the distributions are just advected differently.
The first two steps can be combined as the collide step and represent the computationally intensive part. Usually, implementations choose a two lattice strategy for the update, which eliminates all data dependencies between last and current time step. Note that this doubles memory consumption but simplifies the implementation.

\section{GPGPUs and their programming paradigms}

Test environment. All tests have been performed with the CUDA 4.0 Toolkit and the Intel C Compiler Version 12.0 using the highest optimization level, while maintaining numerically correct results.

The Intel Westmere EP platform used here accommodates two sockets, each holding a 6-core processor (Intel Xeon X5650) running at $2.66 \mathrm{GHz}$ (max. turbo frequency $3.06 \mathrm{GHz}$ ) and having $12 \mathrm{MB}$ L3 cache. Each processor chip forms a ccNUMA (cache coherent nonuniform memory access) locality domain (LD) since it operates on a single on-chip memory controller.

The important details of all evaluated GPUs can be seen in Tab. 1 and the following section describes the NVIDIA GPU hardware with the necessary details.

Global memory. The global memory serves as the random access memory well known from CPUs and is today an order of magnitude smaller than on most CPU based servers, i.e. 3 to $6 \mathrm{~GB}$ on the current Tesla generation (C2050/C2070). The global GPU memory delivers more than two times the bandwidth, i.e. about $91 \mathrm{~GB} / \mathrm{s}$ sustained with ECC enabled ( $144 \mathrm{~GB} / \mathrm{s}$ w/o ECC according to spec sheet) in comparison to a current standard Intel Westmere based dual socket server. ECC stands for Error Checking and Correction and detects and corrects single bit memory errors. Note that only NVIDIA Tesla cards starting with the GF100/Fermi generation have ECC protection. AMD offers up to 2 GB of memory on the HD 6970 GPU. An unvectorized STREAM copy sustains a memory bandwidth of $110 \mathrm{~GB} / \mathrm{s}$, the vectorized implementation sustains $136 \mathrm{~GB} / \mathrm{s}$ (176 GB/s w/o ECC according to spec sheet).

Multiprocessor. A NVIDIA multiprocessor (MP) consists of several cores which are driven in a single instruction multiple data (SIMD) manner. Due to the threading model on the GPU this is also called single instruction multiple thread (SIMT) model. In general all cores in a SIMD unit are controlled by a simple instruction scheduler and have to do exactly the same instructions, i.e. addition, subtraction, multiplication or 
division, at the same time but on different data. Furthermore, the instruction scheduler cannot alter the sequence of computations predetermined by the compiler in this strict in-order architecture. In contrast to CPU hardware there is no large explicit cache on the GPUs. Newer generations have up to $768 \mathrm{~KB}$ of cache. The shared memory on a GPU is a distinctive feature. In terms of access latency and transfer speed it is comparable to registers, but it can be accessed explicitly, similar to the local memory on Cell Broadband Engine [10]. Hence, there will be no automatic caching but the implementation has to manage data copying to and from the shared memory explicitly. On the one hand this gives more opportunities for optimizations. On the other hand an automatic cache would give the same improvement, in most cases. For static data, there is also a memory feature called "constant memory", which is read only but cached. The distinct differences between major multiprocessor generations can be seen in Tab.1.

SIMD block. The AMD equivalent to a MP is called SIMD block and consists of several stream processors. Each stream processor has four units to process integer and single precision (SP) floating point operations at the same time, programmed by a VLIW (very long instruction word). In case of double precision (DP), all these units are utilized together. So a direct comparison of floating point peak performance between NVIDIA and AMD depends on the choice of precision [11]. Further specifications like cache architecture of the AMD hardware are undisclosed and not the focus of this paper.

Host to device interface. The host to device interface is currently PCIe Gen2 which has a maximum transfer bandwidth of $16 \mathrm{~GB} / \mathrm{s}$ (according to the specifications) between host and device. Although transfers over this bus are not covered in this work, it is well known that the PCIe bus represents one of the major bottlenecks in GPU computing [12].

\subsection{CUDA software environment}

The compute unified device architecture (CUDA) was the first approach to GPGPU computing without the hassle to mimic arithmetic operations as graphical operations. Each task is split up into a host (CPU) and device (GPU) part, the latter is also called kernel. The underlying programming language is $\mathrm{C}$, which got extended by several keywords. The NVCC (NVIDIA CCompiler) understands these extensions and compiles a GPU capable executable. NVIDIA $\mathrm{C}++$ extensions are not discussed in this work.
Explicit kernel calls are necessary to invoke GPU computations. Required parameters, the number of blocks and the number of threads, are specified in this call. The finest level of parallelism is a GPU thread. Each thread is assigned to one specific block. The execution of threads is scheduled in packages called "warps", each comprising 32 threads. For the current GF100 32 threads start simultaneously. As a general guideline the minimum feasible number of threads to schedule per block is 32 and larger thread numbers should be a multiple of that. The programmer has to distribute the work dependent on the threadID and blockID of the current thread. No automatic work sharing construct (e.g., for loops as known from OpenMP [13] ) is implemented. On the G80 architecture one single blocking kernel at a time was issued and the runtime system returned back to the CPU process after the kernel was finished. Starting with CUDA 3.2 and compute capability 2.0 one can issue multiple non-blocking kernels in parallel, which actually get executed in parallel by utilizing different so called GPU streams. Inside a stream, kernel calls and memory copies are kept in the order of their submissions. Tasks of different streams can share the same GPU and overlap work. The runtime system decides on how to best place work items. This gives opportunity for algorithms with low parallelism to perform concurrently with other tasks or to overlap communication and CPU to GPU data copy. Note that overlapping most likely does not happen for kernels, where a single kernel already utilizes all GPU resources. For more details see [14].

\subsection{OpenCL}

The Open Compute Language (OpenCL) [15] (maintained by the a consortium of different hard- and software vendors, e.g. NVIDIA and AMD called KHRONOS GROUP) is currently the most comprehensive approach to simplify and generalize access to compute capabilities offered by GPUs, CPUs and other present and future accelerators. Like CUDA, OpenCL programs can be written in $\mathrm{C}$ and $\mathrm{C}++$ like syntax. Although the basic terminology is different, e.g. a CUDA block is a Workgroup and a CUDA thread is a Workitem, the guidelines for programming OpenCL and CUDA on the same hardware are identical or at least very similar.

In this paper we focus on the existing CUDA architecture and adhere to the CUDA nomenclature and definitions. 


\begin{tabular}{|l|l|l|l|l|}
\hline GPU Board & 8800 GTX & Tesla C1060 & Tesla C2070 & AMD 6970 \\
\hline Architecture & G80 & GT200 & GF100 & Cayman \\
\hline Multiprocessors & 16 & 30 & 14 & 24 \\
\hline Total Cores & 128 & 240 & 448 & 1536 \\
\hline Compute Capability & 1.0 & 1.3 & 2.0 & - \\
\hline Memory [GB] & 0.768 & 4 & 6 & 2 \\
\hline Memory BW [GB/s] & 86.4 & 102.4 & 140 & 176 \\
\hline Peak FP SP [GFLOP/s] & 518 & 933 & 1030 & 2700 \\
\hline Peak FP DP [GFLOP/s] & N/A & 77 & 515 & 683 \\
\hline \hline \multicolumn{5}{|l|}{} \\
\hline Multiprocessor: & 8 & 8 & 32 & 16 \\
\hline Cores & 1024 & 1024 & 1536 & 256 \\
\hline Maximum \# thread & 1024 & 768 & $*$ \\
\hline L2 Cache [kB] & $*$ & $*$ & $16 / 48$ & $*$ \\
\hline Shared memory [kB] & 16 & 16 & $48 / 16$ & $*$ \\
\hline Texture Cache [kB] & 16 & 16 & 32768 & $*$ \\
\hline Register & 8192 & 16384 &
\end{tabular}

Table 1: Major NVIDIA GPGPU multiprocessor revisions with documented features. The size of shared memory and texture cache are adjustable on the GF100. AMD specifications are provided where applicable. $(*$ Feature not documented.)

\subsection{Massively parallel threaded execution}

Access to the global device memory has much higher hardware latencies than known from main memory access on CPUs. Mainly the absence of mechanisms to hide latencies, i.e. caches, hardware prefetching algorithms, rescheduling of the compiled instructions (out of order scheduling on CPUs) are the reasons for this on GPUs. Massive thread oversubscription is the way to hide the latency on GPUs. Threads that are waiting for memory IO, basically a bubble in the execution pipeline, are interleaved with those which have work scheduled. The instruction scheduler unit can switch between warps of blocks very efficiently and instantly, thus, filling these bubbles in the execution pipeline and data pipeline. This architectural feature compensates the high latency of the memory system. Currently a two socket server has up to 24 cores available. On an NVIDIA GPU we now have 448 cores, so in general at least 448 threads are necessary to utilize all cores. On CPUs one or at most two (Simultaneous Multi Threading) high demand processes or threads are usually scheduled per core to minimize costly process or thread switching and migrations. In contrast to that much more threads can be issued on a GPU per hardware core without a drawback in performance. In general a given problem has to be decomposed into at least $1536 * 14=21504$ independent threads to reach the maximum number of concurrently scheduled threads.

The fast scheduling of different threads and warps comes at a price. All resource constraints of threads

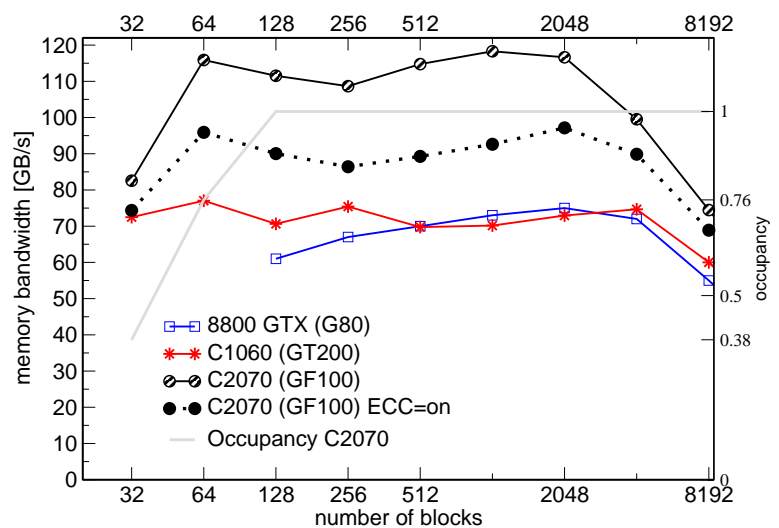

Figure 1: Overview of performance of STREAM copy measurements on different NVIDIA GPU generations for a vector size of $2^{20}$ and 256 threads per CUDA threadblock. 


\begin{tabular}{|l|c|c|c|c|c|}
\hline Threads & 1536 & 1024 & 512 & 256 & 64 \\
\hline GF100 & 20 & 30 & 62 & 125 & 500 \\
\hline GT200 & - & 64 & 32 & 64 & 256 \\
\hline G80 & - & - & 16 & 32 & 128 \\
\hline
\end{tabular}

Table 2: Number of registers available per Thread.

on the MP have to be satisfied right from the start till one block is completely finished. In particular all active threads share the available registers and shared memory on one MP (see Tab. 2). Only 20 registers are accessible for each thread at full occupancy to execute the kernel. Occupancy is defined by the number of actual concurrently running threads divided by the maximum schedulable number of threads.

Increasing kernel concurrency is not sufficient to ensure high performance for memory access. In addition one has to keep in mind coalesced loads and stores and implement the memory access accordingly. Basically threads executed concurrently on a MP need to access data with high spatial locality to get memory requests bundled. This way, latency has to be paid only once for each contiguous memory block. The first G80 based architecture required a strict mapping between thread index and data index. Each thread had to access the element with its thread index starting at an 128 byte aligned address. GT200 introduced caches [16], which relaxed the thread to data mapping substantially. High memory bandwidth can be attained as long as the concurrent data accesses of all active threads have a high spatial data locality. Still an improvement can be seen from correct coalescing. The latest GF100 architecture improved scattered data access even more and only marginal improvements can be seen by correct coalescing.

\subsection{Case study STREAM benchmarks}

The attainable memory bandwidth of GPUs and CPUs can be determined with the STREAM benchmarks [17]. The STREAM copy $\mathrm{C}=\mathrm{A}$, where $\mathrm{A}$ and $\mathrm{C}$ are large vectors, mimics the load to store ratio of the LBM method.

$G P U$. Measurements for all NVIDIA GPGPU generations can be seen in Fig. 1. Note that 8800 GTX is a consumer card. The Tesla series cards are clocked lower than consumer cards of the same generation. The strong scaling of a vector with $2^{20}$ elements with different numbers of blocks and 256 threads per block shows that the scheduling system works very efficiently. We see a lower performance for only 32 blocks as the MPs run only with suboptimal occupancy on C2070. Once occupancy reaches at least 0.5 we see peak memory performance. At 8192 blocks we observe a performance reduction, which correlates to $8192 * 256=2 * 2^{20}$. So half of all threads run empty, which of course impacts performance. The behavior is consistent over all architectures. For larger problem sizes, the performance breakdown will shift accordingly, and occur at larger block sizes.

The exact implementation of ECC on NVIDIA cards is not publicly available. In general ECC [18] is implemented in such a way that for 8 Bytes one additional Byte is stored with redundancy information, i.e. $12.5 \%$ overhead. This is supported by our STREAM measurements as ECC has $10 \%$ to $18 \%$ less user data bandwidth. Note that ECC data reduces the total amount of available space for user data on the GPU (e.g. 5.25 GB instead of $6 \mathrm{~GB}$, which corresponds to $12.5 \%$ redundancy). Furthermore, ECC checksums are calculated in the same arithmetic units as user data and transfered over the same memory subsystem. So immanently, any change of the ECC state changes the data alignment and access pattern as well.

CPU. The OpenMP parallelized STREAM benchmark performance is shown in Tab. 3 and is about $40 \%$ of the GPU bandwidth with ECC. Note that ECC on this server cannot be disabled.

\begin{tabular}{l|ccc} 
& 1 core & 1 NUMA LD & 1 node \\
\hline $\begin{array}{l}\text { Intel X5650 } \\
\text { measured [GB/s] }\end{array}$ & 10.01 & 14.08 & 26.8 \\
$\begin{array}{l}\text { Intel X5650 } \\
\text { actual [GB/s] }\end{array}$ & 15.01 & 21.12 & 40.2
\end{tabular}

Table 3: STREAM copy performance in GB/s. The actual bandwidth accounts for the write allocate.

\section{Performance model}

For GPU and CPU implementations of the LBM the first major performance concern is the memory bandwidth, owing to the computational balance of the LBM of roughly 1.5 Bytes per FLOP in DP [19]. In contrast to that an NVIDIA C2070 has a system balance of 0.27 Bytes/FLOP and an Intel Xeon X5650 dual socket node has 0.16 Bytes/FLOP.

Hence, based on the attainable memory bandwidth one can establish a basic performance model for CPUs and GPUs. For the LBM we have to consider 19 distributions being loaded from and stored to memory. The 
bytes transferred for each LBM lattice cell update can be determined by:

$$
n_{\text {bytes }}=n_{\text {stencil }} \cdot\left(n_{\text {loads }}+n_{\text {store }}\right) \cdot s_{P D F} \text {, }
$$

where $n_{\text {stencil }}$ is the size of the LBM stencil, $n_{\text {loads }}$ and $n_{\text {stores }}$ are the number of loads and stores and $s_{P D F}$ is the size in bytes of a single PDF variable. A peculiarity of cache based architectures like CPUs is that a store to a memory location requires this data to be in the cache. Otherwise a "write allocate" is necessary to fetch the data to the cache. A two lattice implementation leads to 3 total memory transfers on CPUs and to a total of 228 Bytes using single precision and 456 Bytes using DP. On the GPU a total of 152 Bytes using single precision and 304 Bytes using double precision is transferred for a single lattice cell update. The performance of LBM codes is usually given in terms of million fluid lattice cell updates per second (MFLUP/s) instead of GFLOP/s as it is not a feasible performance metric for LBM. The maximum sustainable performance can be derived by dividing the bandwidth obtained from the STREAM benchmarks in Tab. 3 and Fig. 11 by the number of Bytes needed for a lattice update.

Tab. 4 gives the upper performance estimates based on the attainable STREAM bandwidth for the lattice Boltzmann method on the presented architectures.

\begin{tabular}{l|rr} 
& SP & DP \\
\hline Intel X5650 node & 176 & 88 \\
C2070 & 788 & 394 \\
C2070 ECC & 624 & 312 \\
C1060 & 512 & 256 \\
G80 & 492 & N/A
\end{tabular}

Table 4: Performance model for CPU and GPUs in MFLUP/s based on the STREAM bandwidth measurements.

\section{The WaLBerla framework}

WaLBerla [20] is a massively parallel multiphysics software framework that is originally centered around the LBM, but whose applicability is not limited to this algorithm. Its main design goals are to provide excellent application performance across a wide range of computing platforms and the easy integration of new functionality. In this context additional functionality can either extend the framework for new simulation tasks, or optimize existing algorithms by adding specialpurpose hardware-dependent kernels or new concepts such as load balancing strategies. Despite the overhead of a large framework, WaLBerla proved to be as fast as standalone kernels [21]. Various complex simulation tasks have already been incorporated into WaLBerla. Amongst others, free-surface flows [22] particulate flows for several million volumetric particles [23] on up to around 3000000 cores have been included.

\section{Lattice Boltzmann method implementation}

GPU. The application provides sufficient parallelism on the GPU as a single thread computes the update of a single lattice node. The stream-collide sequence was chosen in contrast to [12]. Instead of the local PDFs the collision is computed based on the PDFs of the surrounding lattice cells. The updated PDFs are then stored to the local lattice cell. Hence, the algorithm loads scattered data from the memory, which however, seems to have only very low impact on coalescing and performance. Furthermore, the available data caches support the scattered memory load if the data locality of all threads on the MP is high. The store to main memory happens only at the local cell index and is perfectly coalescable. Note that two grids ensure that no data dependency is violated.

Since, the local stores are aligned it is obsolete to reroute write access through shared memory. Loading coalesced data manually and storing it temporarily in shared memory for later access proved not to be advantageous.

As mentioned above a minimal occupancy of 0.5 is needed to achieve high memory bandwidths. With $2 \times 19$ distribution functions this limit is rather low since the initial implementation led to 100 registers being used in the kernel. Hence, techniques like arithmetic optimizations and minimizing temporary variables have to be employed. Again, storing intermediate data temporarily in the shared memory was not advantageous.

An optimized index operator decreased the register usage down to 32 registers. This technique can be applied to any algorithm working on multidimensional data.

In order to load data perfectly coalesced it has to be aligned to 128 Byte. However, domainsizes are often not obeying this alignment constraint and padding cells are introduced after each stripe of elements. The padding size has to be chosen in such a way, that the next simulation cell starts at an aligned address. Furthermore, the algorithm has to be altered to not compute on the padding elements. 


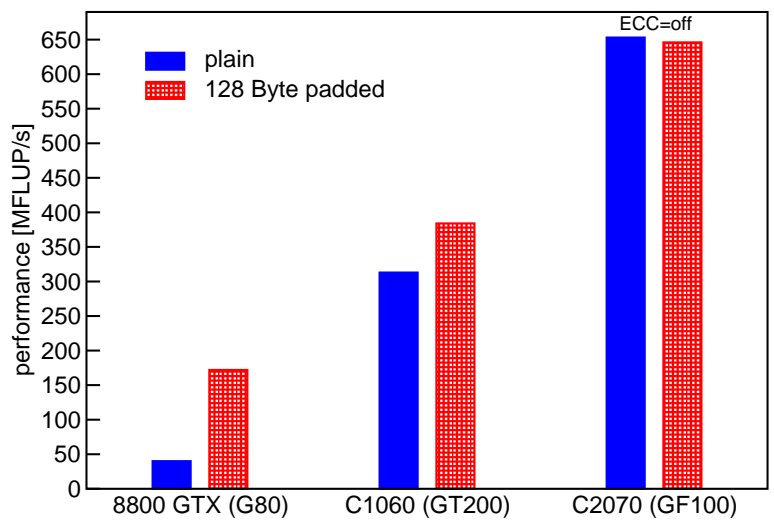

Figure 2: LBM Performance on different NVIDIA GPGPU generations in single precision with a domainsize of $200^{3}\left(158^{3}\right.$ on 8800 GTX owing to limited global memory). Padding introduces overhead cells after each stripe to ensure correct alignment.

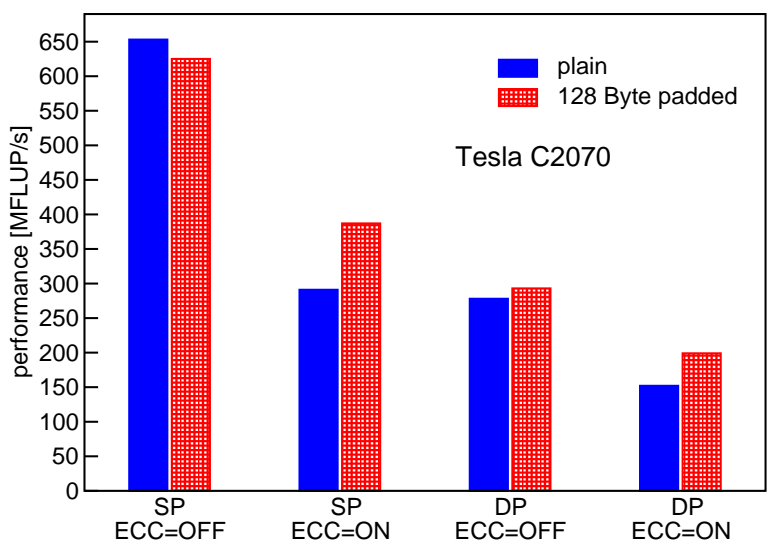

Figure 3: LBM Performance with and without ECC for single and double precision on $\mathrm{C} 2070$ (GF100) with a domainsize of $200^{3}$. For ECC enabled setups and simulations in double precision, padding is beneficial. Only for single precision without ECC, padding has a small negative effect.

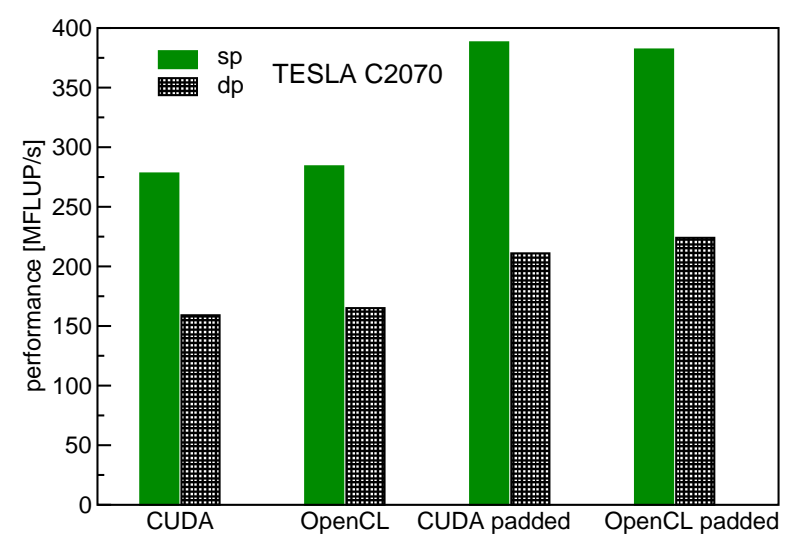

Figure 4: LBM Performance of CUDA and OpenCL implementation on C2070 with ECC with a domain size of $200^{3}$.

\section{Performance results}

The optimized implementation uses one kernel routine for the stream-collide step, but in contrast to general CPU implementations [24], the treatment of non-fluid cells is done in separate kernel. A simple lid-driven cavity problem on different cubic domains was employed to verify the optimized CUDA and OpenCL implementations.

CUDA. The performance of our LBM implementation can be seen in Fig.2 for single precision (SP) ( DP is not supported on the first generation.) and ECC disabled to allow for a comparison with older GPU generations.

We see a two fold speedup from 8800 GTX to C1060, although the memory bandwidth has improved only marginally. The performance gain is mainly due to doubling the available register space on GT200. Consequently, occupancy is twice as before and the sustainable memory bandwidth doubles for our LBM implementation.

Obviously, correct alignment and padded memory access was mandatory on G80 and favorably on GT200 based GPUs. Padding is no longer relevant on GF100 based GPUs for single precision calculations if ECC is disabled. Instead it is counterproductive as additional memory transfers decrease performance slightly. Overall improvement from C1060 to C2070 is about $40 \%$ which corresponds to the increase in memory bandwidth.

For double precision and ECC enabled the influence of padding is as anticipated on C2070 as well. Fig. 3 shows that ECC enabled setups benefit from appropriate padding. Note, that already 16 Byte padded access to memory increases performance but 128 Byte padding gives best performance.

The large performance gap between ECC and nonECC simulations cannot be explained solely by the additional data transfers (see above). This issue here is currently under investigation.

OpenCL GPU. Next we compare the performance of our CUDA implementation to an OpenCL implementation. The CUDA kernels needed minimal adjustments to work under OpenCL. Basically the calculation of the local index by taking CUDA threadID and blockID were replaced by OpenCL's localID and groupID. The remaining kernel adjustments affect only function declarations. The performance of OpenCL in comparison to CUDA can be seen in Fig. 4. Qualitatively speaking there is no difference in ECC enabled setups for single and double precision. Padding has the same positive influence as for CUDA. 

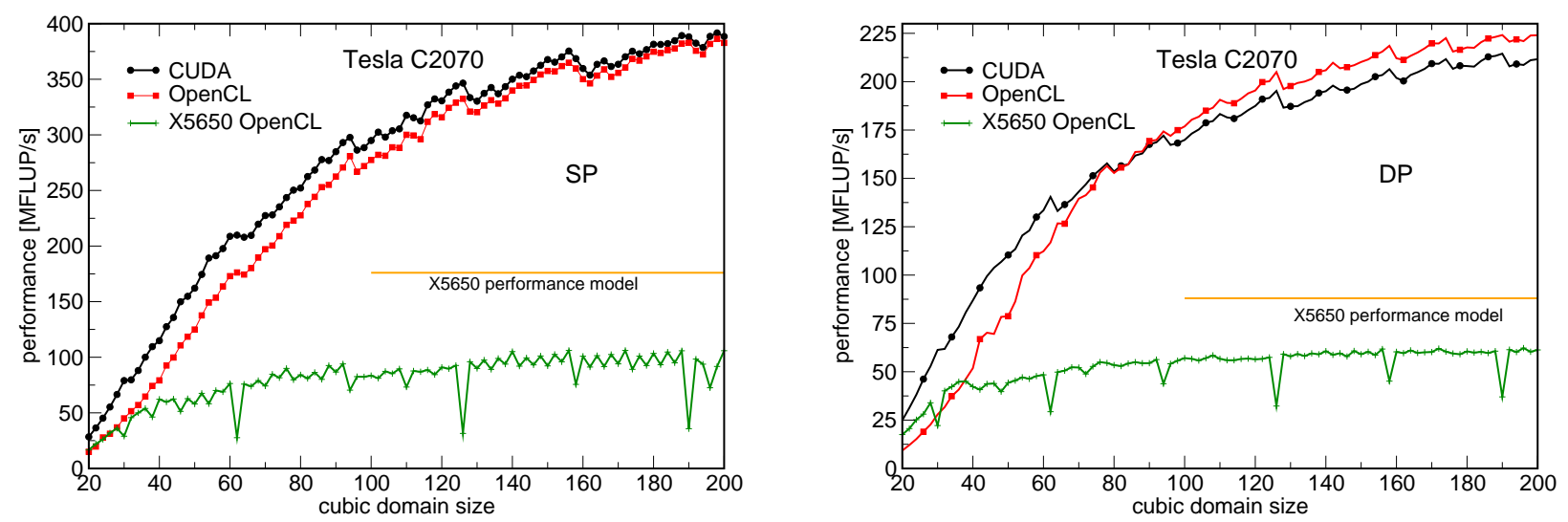

Figure 5: Performance comparison of CUDA and OpenCL on C2070 with padding in SP (upper) and DP (lower) with ECC. Furthermore, measurements with the same OpenCL implementation on the Intel Xeon X5650 CPU are shown sustaining about $60 \%$ of the performance model in SP

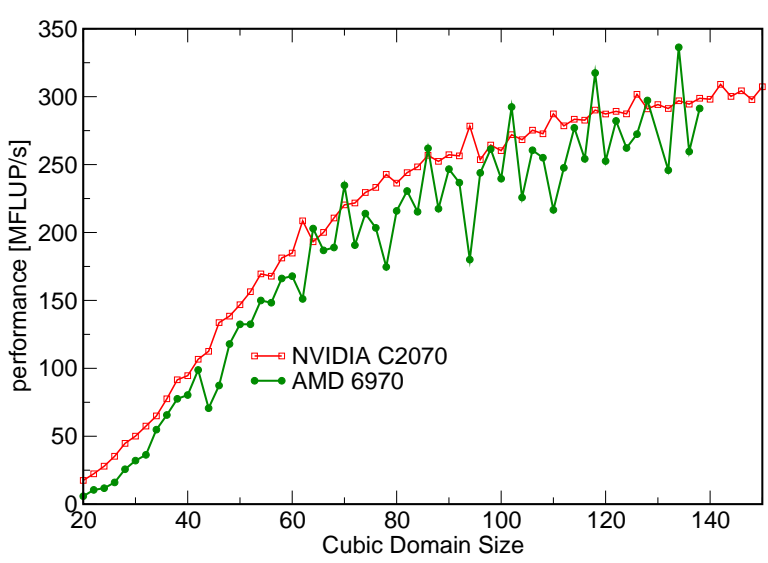

Figure 6: Performance comparison of AMD 6970 and NVIDIA Tesla C2070 with OpenCL in DP without ECC.

A full domain sweep is shown in Fig. 5 for SP and DP. In SP OpenCL is on par with CUDA on the GPU. For DP OpenCL is indeed faster by $6 \%$ for large domains. Both algorithms show the same overall behavior, although CUDA is faster for domains up to $80^{3}$. The small peaks occurring at domain sizes of multiples of 16 are perfectly aligned and no padding needs to be applied. Benchmarks for the OpenCL enabled AMD HD 6970 can be seen in Fig. 6, compared to the results of the NVIDIA C2070. Overall performance is comparable to the $\mathrm{C} 2070$ performance but more erratic. A possible reason is the missing cache hierarchy which eases latency hiding on the C2070. Based on the memory bandwidth of the spec sheet one would anticipate a $19 \%$ higher performance on HD 6970. However, our code was thoroughly optimized and tested for CUDA and
NVIDIA GPUs, but not for OpenCL and AMD based GPUs. These results are very encouraging for further GPGPU development as OpenCL has caught up with CUDA and provides access to more versatile hardware and even CPUs.

OpenCL CPU. Measurements of OpenCL on the CPU are shown in Fig.5]as well. Comparing the DP CPU performance to the performance model we obtain $71 \%$ of performance on this architecture. So there is still room for improvement. For SP we do not see twice the performance as in DP in contrast to our estimates of the performance model in Tab. 4. Obviously, memory bandwidth is not the issue here. A look at the performance counters for vectorized and scalar FLOPS with likwid [25, 26] shows just scalar operations. Thus, only a fourth of the possible SP FLOPS of the Intel XEON can be utilized.

\section{Conclusion}

We here presented a highly optimized LBM based kernel for GPGPUs in CUDA and OpenCL. Since the LBM is mainly memory bandwidth bound and a single lattice update takes 304 Byte on the GPU and 456 Byte on the CPU in DP, we initially established an upper performance limit based on sustainable memory bandwidth by implementing benchmarks for basic memory operations, the STREAM benchmarks. Up to $110 \mathrm{~GB} / \mathrm{s}$ can be sustained on a NVIDIA C2070 GPU (95 GB/s with ECC enabled) and up to $40 \mathrm{~GB} / \mathrm{s}$ on the Intel XEON X5650 host node. The usage of ECC shows a loss of $10 \%$ to $18 \%$ on the GPU which is close to the limit of 
$12 \%$ suggested by the mostly undisclosed documentation and the bandwidth overhead.

The same LBM kernel structure is applied in both CUDA and OpenCL and leads to $83 \%$ of the sustainable memory bandwidth in single precision without ECC memory. Enabling ECC leads to a substantial loss in performance between 30 and $40 \%$. To determine the reason for this discrepancy is the topic of current research. However, using ECC is inevitably necessary to ensure correct simulation results and should not be neglected to favor faster execution. Only NVIDIA Tesla cards currently support ECC memory. Still the GPU kernels give a speedup of two in contrast to a full two socket Intel Xeon server with optimized C intrinsics [24].

The implemented OpenCL kernel performs well on both, NVIDIA and AMD GPUs with the same performance. Furthermore, the performance is on par with the CUDA measurements.

The OpenCL GPU kernel performs surprisingly well on the CPU, which reaches $71 \%$ of the performance model. These are first promising results, as no optimizations for the CPU have been applied. A speedup of less than two from DP to SP and the absence of any packed instructions leads to the conclusion that the OpenCL compiler is not capable of vectorizing the code.

\section{Acknowledgements}

This work was partially funded by the "Bundesministerium für Bildung und Forschung" under the $S K A L B$ project, no. 01IH08003A, and by the "Kompetenznetzwerk für Technisch-Wissenschaftliches Hochund Höchstleistungsrechnen in Bayern" (KONWIHR) via OMI4papps. The authors thank Fujitsu for providing the "Sandy Bridge" platform through an early access program.

\section{References}

[1] Li W, Wei X, Kaufman A. Implementing lattice boltzmann computation on graphics hardware. The Visual Computer 2003;19:444-56. URL http://dx.doi.org/10.1007/ s00371-003-0210-6.

[2] Tölke J, Krafczyk M. Teraflop computing on a desktop pc with gpus for $3 \mathrm{~d}$ cfd. International Journal of Computational Fluid Dynamics 2008;22(7):443-56.

[3] Obrecht C, Kuznik F, Tourancheau B, Roux JJ. A new approach to the lattice boltzmann method for graphics processing units. Computers \& Mathematics with Applications 2011;61(12):3628-38.

[4] Obrecht C, Kuznik F, Tourancheau B, Roux JJ. Multi-gpu implementation of a hybrid thermal lattice Boltzmann solver using the TheLMA framework. In: Proceedings of the 23st International Conference on Parallel Computational Fluid Dynamics. Parallel CFD 2011; 2011,
[5] Wolf-Gladrow DA. Lattice-Gas Cellular Automata and Lattice Boltzmann Models; vol. 1725 of Lecture Notes in Mathematics. Berlin: Springer; 2000.

[6] Succi S. The Lattice Boltzmann Equation - For Fluid Dynamics and Beyond. Clarendon Press; 2001.

[7] Chen S, Doolen GD. Lattice Boltzmann method for fluid flows. Annu Rev Fluid Mech 1998;30:329-64.

[8] Qian YH, d'Humières D, Lallemand P. Lattice BGK models for Navier-Stokes equation. Europhys Lett 1992;17(6):479-84.

[9] Ziegler DP. Boundary conditions for lattice Boltzmann simulations. J Stat Phys 1993;71(5/6):1171-7.

[10] The Cell Broadband Engine Architecture, Version 1.02. http://www.ibm.com/developerworks/power/cell/ documents.html; 2007.

[11] AMD 6970 GPU. http://www.amd.com/us/products/ desktop/graphics/amd-radeon-hd-6000/hd-6970/; 2011.

[12] Habich J, Zeiser T, Hager G, Wellein G. Performance analysis and optimization strategies for a D3Q19 lattice Boltzmann kernel on nVIDIA GPUs using CUDA. Advances in Engineering Software 2011;42(5):266 -72. URL http://www.sciencedirect.com/science/article/ pii/S0965997810001274.

[13] Open MP. http://www.openmp.org/; 2011.

[14] nVIDIA Cuda Programming Guide 4.0. http://developer. download.nvidia.com/compute/DevZone/docs/html/ C/doc/CUDA_C_Programming_Guide.pdf; 2011.

[15] KHRONOS GROUP (TM) OpenCL Specification. http:// www.khronos.org/opencl/; 2011.

[16] Volkov V, Demmel JW. Benchmarking GPUs to tune dense linear algebra. In: Proceedings of the 2008 ACM/IEEE conference on Supercomputing. SC '08; Piscataway, NJ, USA: IEEE Press. ISBN 978-1-4244-2835-9; 2008, p. 31:1-31:11. URL http: // portal.acm.org/citation. cfm?id=1413370.1413402.

[17] The Stream benchmark. http://www.streambench.org/; 2011.

[18] Wikipedia ECC-Memory. http://en.wikipedia.org/ wiki/ECC_memory; 2011.

[19] Wellein G, Zeiser T, Hager G, Donath S. On the single processor performance of simple lattice Boltzmann kernels. Computers \& Fluids 2006;35(8-9):910-9.

[20] Feichtinger C, Donath S, Köstler H, Götz J, Rüde U. Walberla: HPC software design for computational engineering simulations. Journal of Computational Science 2011; In Press,(2008). URL http://linkinghub.elsevier.com/ retrieve/pii/S1877750311000111

[21] Feichtinger C, Habich J, Köstler H, Hager G, Rüde U, Wellein G. A flexible patch-based lattice Boltzmann parallelization approach for heterogeneous GPU-CPU clusters. Parallel Computing 2011;37(9):536 -49. URL http: //www. sciencedirect . com/science/article/pii/S0167819111000342.

[22] Donath S, Mecke K, Rabha S, Buwa V, Rüde U. Verification of Surface Tension in the Parallel Free Surface Lattice Boltzmann Method in WaLBerla. Computers \& Fluids 2011;2(2):105 -12.

[23] Götz J, Iglberger K, Stürmer M, Rüde U. Direct numerical simulation of particulate flows on 294912 processor cores. IEEE computer society (Veranst): 2010 ACM/IEEE International Conference for High Performance Computing, Networking, Storage and Analysis (Supercomputing 2010, New Orleans, 1311 - 19112010) 2010;:1 - 11.

[24] Wellein G, Habich J, Hager G, Zeiser T. Node-level performance of the lattice Boltzmann method on recent multicore CPUs. In: Proceedings of the 23st International Conference on Parallel Computational Fluid Dynamics. Parallel CFD 2011; 2011,. 
[25] Treibig J, Hager G, Wellein G. Likwid: A lightweight performance-oriented tool suite for x 86 multicore environments. Parallel Processing Workshops, International Conference on Parallel Processing Workshops 2010;0:207-16.

[26] LIKWID. http://code.google.com/p/likwid; 2011. 